\title{
Il fumo di "cannabis legale" come strumento per la riduzione del danno alla salute da tabacco
}

\author{
Fulvio Fantozzi*
}

\begin{abstract}
SUMMARY
- The Author deals with a new therapeutic tool aimed to cut down tobacco health problems i.e. cannabis which has got a very slow THC content and therefore no narcotic effect. Of course, since that cannabis does not contain nicotine at all, people smoking such stuff everyday will never become nicotine addicts eventually. He puts his evaluation inside the conceptual frame of latest FDA policy about tobacco health problems in the US i.e. the eradication of compulsion for cigarettes as the number one goal and the diffusion of low nicotine content (or better no nicotine content) cigarettes as the mean to achieve it. He argues that first of all we should be sure there is no risk to get THC-positive urine samples from people who will smoke or heat such cannabis products and tells of his personal self experimentation about that issue.

Keywords: Smokable cannabis and tobacco, E-cig, Tobacco harm reduction, Self-experimentation.

Parole chiave: Cannabis e tabacco combustibili, Sigaretta elettronica, Riduzione del danno da tabacco, Autosperimentazione.
\end{abstract}

ATTENZIONE: si consiglia di non provare a replicare a casa vostra quanto descritto qui di seguito nel paragrafo "AUTOESPERIMENTO".

A mio parere è estremamente interessante osservare come gli USA si sono mossi nel corrente anno 2017 in termini di pronunciamenti su tabacco e salute pubblica.

Il nuovo (dis)corso statunitense è complesso ed affonda le proprie radici ovviamente in un lavoro attento di revisione e di progettazione di interventi di sanità pubblica già decollato alcuni anni fa sotto l'egida della FDA.

Qui riporto in rapida sintesi i contenuti di due documenti recenti e a mio avviso molto rilevanti sul tema:

1. I'ultimo pronunciamento antifumo della FDA (1), imperniato sulla difesa dei giovani americani esposti a morte prematura a causa del tabacco.

Il "cuore" del documento è costituito a mio giudizio dalla seguente affermazione: "il punto nodale della strategia per affrontare la crisi di dipendenza da tabacco nelle nuove generazioni che sta minacciando le famiglie americane è modificare $i$ livelli di nicotina nel fumo derivante da combustione di sigarette".

2. un documento pubblicato il 21 settembre scorso sulla prestigiosa Rivista medica New England Journal of Medicine (NEJM) (2) dal dr Scott Gottlieb, lo stesso Responsabile della FDA che aveva firmato il citato pronunciamento il 28 luglio scorso (1). I punti salienti dell'articolo sono altri, sebbene l'attenzione al

\footnotetext{
* Medico delle Dipendenze e Tabaccologo libero professionista. Specialista in Medicina Legale e delle Assicurazioni e Perfezionato in Dipendenze Patologiche, Tossicologia Clinica, Bioetica e Psicopatologia Forense, già Dirigente Medico di Farmacologia e Tossicologia Clinica e Direttore dei Centri Antifumo di Carpi e Mirandola della Az. USL di Modena.
}

rischio di danno alla salute da tabacco delle nuove generazioni sia ancora ben presente. Ne riportiamo alcuni:

- la riduzione del consumo di sigarette combustibili (il riferimento implicito non è tanto alla sigaretta elettronica, abbreviata in "e-cig", la quale non provoca "combustione", ma alle sigarette che erogano fumo a seguito di riscaldamento di sticks di tabacco, come la ben nota IQOS);

- la riduzione della dipendenza dalle sigarette di tabacco mediante l'abbattimento del loro contenuto in nicotina non solo a tutela dei giovani che potrebbero così usarle senza diventare dipendenti, ma per il bene dei fumatori di qualsiasi età e segnatamente per facilitarne la disassuefazione (il concetto è ripetuto tale e quale, come un mantra, due colonne di testo più avanti);

- I'attenzione a non favorire semplicemente il passaggio da una forma di nicotina (le sigarette convenzionali) ad una qualsiasi altra forma di dispensazione di nicotina in soggetti dipendenti;

- la cautela nel non favorire involontariamente con azioni di regolazione del mercato l'emergere di rimbalzo di un mercato clandestino di sigarette ad alto contenuto di nicotina.

Ma lo slogan che si ricava dalla lettura attenta dell'articolo è che si dovrebbero fare due cose se si vuole davvero promuovere la riduzione del danno da tabacco: focalizzare gli interventi sulla nicotina e supportare l'innovazione in merito a nuove forme di consumo voluttuario di prodotti fumabili o "svapabili".

Si citano poi articoli scientifici che sostengono la tesi per cui il paventato fenomeno del "fumo compensatorio" di sigarette a basso contenuto di nicotina è una sorta di bufala, si ricorda il ruolo che la NRT vale a dire la terapia sostitutiva con preparati a base di nicotina potrà avere nell'ottica della riduzione del danno e si riconosce il ruolo altrettanto importante delle e-cig in materia, ma d'altro canto la necessità di regolamentarne la vendita. Da ultimo si esplicita il significato della strategia suddetta in questi termini: "i benefici per la salute pubblica derivati dall'implementazione di una politica di riduzione della nicotina nelle sigarette combustibili potrebbero essere enormi". 


\section{E qui in Italia che si fa? E che si pensa?}

A mio avviso sul tema che informa l'articolo del NEJM, ossia la riduzione del danno da tabacco in fumatori inveterati poco motivati a smettere, la sensibilità di chi opera in trincea in Italia è scarsa.

A poco servirono contributi comparsi timidamente nella stampa di settore già 17 anni fa ad opera anche del sottoscritto (3).

Le droghe legali, tabacco e alcol, hanno sempre evocato atteggiamenti radicali del tipo "tutto o nulla" (o smetti del tutto o continui a fare quel che fai) e solo da 2-3 anni in Alcologia sono finalmente comparse e si sono consolidate nella pratica clinica posizioni laiche che ammettono trattamenti orientati alla mera riduzione del bere eccessivo e patologico e non alla sua immediata cessazione, almeno come primo "step" clinico.

Ciò grazie all'impiego oculato di farmaci ad hoc come naltrexone $\mathrm{e}$, in subordine, nalmefene (4).

Per il tabagista le strade sono invece sempre le solite: smettere per i motivati a farlo, lavorare sulla motivazione a smettere per i non ancora motivati a farlo.

Sul versante del tabagismo che affligge pazienti in carico ai Servizi Psichiatrici per malattie mentali croniche gravi si è registrato qui e là (e lo dico dal mio piccolo osservatorio privato di medico delle dipendenze che cura anche tabagisti e tra di loro anche tabagisti con forte comorbidità psichiatrica) un certo interesse all'impiego della NRT allo scopo dichiarato di ridurre il numero di sigarette fumate e quindi il danno da tabacco, senza pretendere di fare smettere "lo psicotico cronico da 60 sigarette al giorno". Purtuttavia non vi sono state prese di posizione istituzionali esplicitamente a favore della diffusione di un approccio del genere nelle strutture sanitarie pubbliche, nemmeno in quelle più avanzate come quelle di Reggio Emilia, nelle quali chi scrive ebbe l'opportunità di lavorare come Medico Tabaccologo Responsabile del primo Centro Antifumo nato in seno al Ser.T. nel lontano 1999. Forse è per via di tale improvvida latitanza del mondo sanitario italiano, ma anche dei "pensatori pubblici" nei confronti della riduzione del danno alla salute del tabagista incallito vale a dire quello/a non ancora pronto/a smettere, che le pagine cartacee e web dei media italiani presentano sempre più spesso dall'inizio del 2017 interventi sensazionali, talora accorati e talora invece misuratamente, ma acutamente critici (5), sui benefici della cannabis legale e su quanto e come essa possa divenire un valido ausilio per chi vuole se non proprio smettere, almeno fumare meno tabacco. In concreto si tratterebbe di sostituire parte delle sigarette convenzionali di tabacco fumate tutti i giorni (o addirittura di rimpiazzarle tutte tout court!) da forti ed impenitenti fumatori, di sostituirle dicevamo, "udite udite"... con sigarette contenenti non tabacco, ma cannabis "legale" laddove il suo contenuto di THC è ben al di sotto al limite di legge attuale dello 0,2\%.

In questa sede non commento le posizioni pro-legalizzazione che a mio avviso si nascondono dietro alla facciata falsamente filantropica e sanitaria di alcune tra le esternazioni sull'argomento, perché questo mio scritto, che pure ha sembianze di pamphlet, non vuole essere affatto politico.

Preciso inoltre che non sono mai stato fumatore e che in merito alle ipotesi di legalizzazione/liberalizzazione delle cosiddette droghe leggere ho sempre espresso pubblicamente la mia posizione motivatamente e non ideologicamente contraria.

"Non ideologico" significa che se un domani qualcuno dimostrasse che per la salute pubblica i "pro" della legalizzazione del THC fumato a scopo ricreativo superano i "contro", io tranquillamente cambierei idea e mi schiererei a favore della legalizzazione suddetta. [Distorsioni cognitive di chi nel THC "c'è dentro fino al collo" ed interessi di bottega di chi cavalca l'onda dell'antiproibizionismo più o meno dichiaratamente solo per partito preso costituiscono notoriamente invincibili fattori di costrizione per chi ha posizioni ideo- logiche in materia, nel senso che costringono costoro a mantenerle salde tali posizioni, insensibili alle dimostrazioni del contrario].

Tanto premesso oggi ragionare di uso terapeutico di preparazioni non solo farmaceutiche a base di cannabis, vale a dire cannabis fumabile a concentrazione di THC inferiore allo 0,2\%, dunque legale, è molto opportuno e molto fondato scientificamente.

\section{La putativa beneficialità del fumo di cannabis legale ovvero le virtù del CBD}

La stampa laica italiana e la pubblicità di chi produce cannabis legale puntano sul cannabidiolo, (CBD) quale componente psicoattiva benefica della cannabis, idonea ad esercitare un effetto curativo sinergico rispetto al THC non solo per le patologie per le quali preparazioni farmaceutiche a base di THC sono già state autorizzate nel nostro Paese, ma anche " da solo", laddove capace anche da solo di "modulare vari circuiti neuronali coinvolti della dipendenza da sostanze" (6). Fino a 2 anni fa (il lavoro appena citato uscì nel 2015 ed esaminò la letteratura scientifica dell'epoca) si poteva affermare che "un numero limitato di studi preclinici (preclinico = su animali, $\mathrm{NdR}$ ) suggerisce che il CBD può avere proprietà terapeutiche sulle dipendenze da oppiacei, cocaina e psicostimolanti ed alcuni dati preliminari suggeriscono che esso può essere efficace nella dipendenza da cannabis e da tabacco nell'Uomo".

Si tratta in effetti di un componente affascinante del fumo di cannabis le cui proprietà essenzialmente antipsicotiche e rilassanti abbinate all'innocuità sul piano addittologico (il CBD è incapace di generare dipendenza) debbono però ancora essere puntualizzate, qualificate e comunque correttamente dimensionate quanto a loro magnitudo e dunque rilevanza farmacologico-clinica.

Un unico studio nell'Uomo, condotto con metodologia pregiata (doppio cieco), ma su di un campione a mio parere poco numeroso e con un periodo di osservazione troppo breve, evidenziò effetti positivi del CBD rispetto al placebo in termini di apprezzabile riduzione del contemporaneo consumo di sigarette di tabacco (7).

\section{La putativa "non maleficialità" del fumo di canna- bis legale ovvero: le cautele da osservare prima di raccomandarne l'uso}

Tornando al presupposto scientifico ed etico di questo articolo, ovvero che nell'ottica della riduzione del danno da tabacco sia cosa buona e giusta ragionare di preparazioni assunte mediante combustione o meglio ancora mero riscaldamento con appositi dispositivi di altre matrici vegetali, tutto porta a pensare alla cannabis legale come ad uno strumento posto egregiamente sulla traiettoria strategica tracciata dai recentissimi documenti USA sopra esaminati $(1,2)$, soprattutto dal secondo: la cannabis legale viene fumata sì, e comporterà pertanto un rischio fisico, forse al pari del tabacco, derivato dalla tossicità di sostanze irritanti, monossido di carbonio e catrame, ma almeno non contiene né concentrazioni rilevanti di THC né per nulla nicotina, dunque non è rispettivamente né stupefacente né dipendentigena!

Un difetto che però si coglie nella trattazione divulgativa dell'argomento è che si dà per scontato che l'uso di trinciati di cannabis legale fumati o riscaldati sia innocuo, prima ancora che sul piano della salute del consumatore abituale, sul piano del rischio di avere grane con la patente e/o col lavoro.

La domanda alla quale a mio parere apoditticamente si fornisce risposta affermativa è: "siamo sicuri che consumare cannabis a basso concentrazione di THC non vada a 'sporcare' le urine facendole divenire positive alle analisi che comunemente sono fatte in materia di droghe e guida e droghe e lavoro?". 
Pare infatti che non vi siano studi sul campo che lo dimostrino. Ebbene espongo qui il mio piccolo contributo sperimentale consistito nel testare su me stesso, ossia un volontario sano di 59 anni, caucasico, in buona salute, peso $83 \mathrm{~kg}$ ed altezza 1,81 cm, non assuntore abituale di farmaci e da sempre non fumatore di tabacco o altre droghe oltre che bevitore occasionale e controllato di bevande alcoliche, una preparazione fumabile di cannabis con contenuto di $\mathrm{THC}<<0,2 \%$ esclusivamente in relazione al parametro della sicurezza per patente e lavoro.

In concreto ho fumato n. 4 sigarette di quel tipo, arrotolate artigianalmente dal produttore con la classica carta da sigaretta.

Probabilmente a spingermi a questa inusuale pratica dello sperimentare su se stessi sono stati l'esempio di geniali psiconauti, come Albert Hofmann, il creatore dell'LSD e le suggestioni potenti di quel grande pioniere contemporaneo che è il Prof. Beniamino Palmieri dell'Università degli Studi di Modena e Reggio Emilia (8).

Prima di raccontare l'esperimento debbo tornare a questioni più prosaiche ed immanenti e precisare che se in teoria si dovrebbero incriminare e condannare conducenti che si sospetta abbiano usato droghe prima di guidare solo se l'esame del loro sangue è risultato positivo ad una concentrazione ancorché minima di quella droga o sostanza psicoattiva, nella pratica purtroppo molti cittadini sono stati e tuttora sono correntemente incriminati e condannati ai sensi dell'art. 187 del Codice della Strada (oppure costretti ad abbandonare la loro mansione lavorativa se I'esame è stato fatto dal Medico Competente d'Azienda ai sensi del D.Lvo 81 del 2008 ) sulla base di un test tossicologico positivo effettuato sulla sola matrice biologica urinaria!

Dunque ancora oggi avere un test urinario positivo per THC assume enorme rilevanza ed enorme perniciosità per un conducente e/o lavoratore.

\section{Auto-esperimento}

Soggetto testato: il sottoscritto.

Trattasi di un volontario sano di età di 59 anni, caucasico, in buona salute, peso $83 \mathrm{~kg}$, altezza 1,81 cm, non assuntore di farmaci e da sempre non fumatore di tabacco o altre droghe oltre che bevitore occasionale congruo e controllato di bevande alcoliche.

In data 19/9/2017 assunzione per via respiratoria e nell'arco di 6 ore del fumo di n. 4 sigarette contenenti cannabis sativa varietà Futura 75 SOC France, $\mathrm{THC}=0,033 \%$.

Inspirazioni profonde del suddetto fumo di durata 3 ", con intervalli di $15^{\prime \prime}$ e trattenimento del fumo nelle vie respiratorie per esattamente $5^{\prime \prime}$. Durata della consumazione di ogni sigaretta: dai $6^{\prime}$ ai $7^{\prime}$.

Misurazione del CO espirato prima della sessione fumatoria: 2 parti per milione ( $\mathrm{ppm})$

Ore 10.00: prima sigaretta, nessun effetto soggettivo né fisico né psichico rilevante.

Ore 11.30: seconda sigaretta, idem.

Ore 14.00: terza sigaretta, idem.

Ore 16.00 quarta sigaretta. Avverto sensazione di tenue bruciore delle fauci. Alle ore 16.07 decido di terminare la sessione auto-sperimentale sia per il timore che la quinta sigaretta induca un vero e proprio disturbo da intolleranza fisica sia perché opino che l'introito di fumo sia sufficiente per l'obiettivo del test che, ricordo, è la prima verifica empirica dell'innocuità della pratica del fumare cannabis legale sul piano dell'esito dei test THC comunemente utilizzati in ambito sanitario e legale (THC e guida e THC e lavoro); a latere misuro il CO espirato, il quale risulta 1 ppm; alle ore 23.45 effettuo una terza ed ultima misurazione del CO espirato, che risulta nuovamente uguale a $1 \mathrm{ppm}$.

Il giorno successivo, esattamente 13 ore e mezzo dopo il consumo delI'ultima sigaretta di cannabis legale, termino l'autosperimentazione eseguendo la determinazione del THC urinario con un test singolo ad immersione e lettura ottica immediata, cut off $=50 \mathrm{ng} / \mathrm{ml}$.

L'esito è negativo, come... "non atteso", nel senso che attendevo un esito positivo dell'esame data la non irrilevanza della quantità di cannabis fumata in un arco temporale piuttosto ristretto (4 ore).

\section{Conclusione}

Il risultato della autosperimentazione sopra descritta incoraggia a mio avviso l'esecuzione di vere e proprie sperimentazioni, da eseguirsi su matrici biologiche adeguate (urine e soprattutto sangue) e su di un numero significativo di volontari sani, finalizzate a convalidare la tesi più volte espressa a mio giudizio in modo poco convincente sulla stampa non medica da persone motivate ed entusiaste sì, ma "non informate dei fatti" nel senso di privi di competenze mediche e biologiche, secondo la quale fumare cannabis legale ossia a contenuto di THC $<0,2 \%$ non espone al rischio di guidare/lavorare in stato di alterazione psichica.

Possiamo per ora affermare solo che la modesta autosperimentazione in parola non contrasta né però conferma l'affermazione apodittica di cui sopra, poiché per dimostrare che le cose stanno davvero così si dovrebbe allestire una sperimentazione "come si deve" da parte di un organo neutrale ed istituzionale (ISS e/o Università), con metodologia incontestabile, campioni numerosi e tutti gli altri crismi del caso.

L'importante è non trascurare, bollandole presuntuosamente come eclettiche o addirittura devianti, questioni come quella sopra discussa.

Al di là di prevedibili per non dire scontate problematiche di ordine legale, autorizzativo, burocratico che ne ostacolerebbero molto probabilmente la sperimentazione nel nostro Paese l'uso terapeutico della cannabis di fatto priva del THC riveste e rivestirà infatti un interesse a mio parere più che apprezzabile anche nel campo della Tabaccologia Medica e segnatamente nella particolare sottopopolazione dei fumatori disponibili e pronti a fumare meno sigarette di tabacco, ma non (ancora) a smettere del tutto e purtroppo non "orientabili" a pratiche sostitutive più convenzionali e "facili "come "svapare" e-cig, riscaldare sticks di tabacco nei dispositivi IQOS o assumere farmaci a base di nicotina (NRT).

Dichiarazione su eventuali conflitti di interessi: l'Autore non ha collaborazioni professionali né con l'Industria del Farmaco né con quella del Tabacco né con produttori di piante medicinali o di dispositivi per l'assunzione voluttuaria di fumi o vapori di sorta, né ha contenziosi in atto con tali Organizzazioni.

\section{Bibliografia}

1. FDA announces comprehensive regulatory plan to shift trajectory of tobacco-related disease, death. For Immediate Release, July 28, 2017 www.fda.gov/NewsEvents/Newsroom/PressAnnouncements/ucm568 923.htm, ultimo accesso 5/11/2017.

2. Gottlieb S., M.D., Zeller M., J.D. A. (2017). Nicotine-Focused Framework for Public Health. N.Engl.Med. J., 377;12, September 21.

3. Fantozzi F. (2000). Tabagismo e riduzione del danno: un approccio originale. Personalità/Dipendenze, 6 (fasc. I).

4. Fantozzi F. (2014). Capitolo "Il ruolo del sistema oppioide: curare antagonizzando", in Alcol: capire e orientare i consumi, diagnosticare e curare abuso e dipendenza, pp. 305-316. Verona: Ed. CLAD ONLUS.

5. Gatti R. La cannabis "light". www.droga.net/la-cannabis-light/, ultimo accesso 5/11/2017.

6. Prud'homme et al. (2015). Cannabidiol as an Intervention for Addictive Behaviors: A Systematic Review of the Evidence. Substance Abuse: Research and Treatment, 9: 33-38. doi: 10.4137/SART.S25081.

7. Morgan C.J., Das R.K., Joye A., Curran H.V., Kamboj S.K. (2013). Cannabidiol reduces cigarette consumption in tobacco smokers: preliminary findings. Addict Behav., 38(9): 2433-6.

8. Palmieri B., Sblendorio V., Palmieri L. Dipartimento di Chirurgia Generale e Specialità Chirurgiche, Università di Modena e Reggio Emilia. L'autosperimentazione in medicina: una filosofia emergente dal network medico-cura-te-stesso. www.networksecondoparere.it/ data/repository/20120175108d5303f/autosperimentazioneinmedicin a.pdf, ultimo accesso 5/11/2017. 